Zusammenfassung der Dissertation mit dem Titel

\title{
„Nebenbestimmungsfeindliche Verwaltungsakte“
}

Dissertation vorgelegt von Sebastian Traub

Erstgutachter: Prof. Dr. Wolfgang Kahl

Zweitgutachter: Prof. Dr. Peter Axer

Institut für deutsches und europäisches Verwaltungsrecht 
Die Dissertation mit dem Titel „Nebenbestimmungsfeindliche Verwaltungsakte“ wurde im Sommersemester 2017 von der Juristischen Fakultät der Universität Heidelberg angenommen. Sie erscheint im Verlag Mohr Siebeck (Tübingen) in der Reihe „Beiträge zum Verwaltungsrecht (BVwR)“ als Band 5; die nachfolgenden Verweise beziehen sich auf die Druckfassung.

I.

Ausgangspunkt der Dissertation - und insoweit nimmt die Einleitung (S. 1-13) hier auch ihren „,natürlichen“ Einstieg - ist der Stand der tradierten Dogmatik (vermeintlich) nebenbestimmungsfeindlicher Verwaltungsakte. Seit der wirkmächtigen Schrift Karl Kormanns „System der rechtsgeschäftlichen Staatsakte“ (1911) orientiert sich die in Rechtsprechung und Literatur vorherrschende Fallgruppenbildung an Verwaltungsaktstypen, bei denen - über fachrechtliche Ausschlussgründe hinaus - die Beifügung von Nebenbestimmungen aufgrund der Natur des Verwaltungsakts ausgeschlossen sein soll. Einen gerafften Überblick liefert bereits die Durchsicht der einschlägigen Lehrbuch- und Kommentarliteratur: Zentrale Beispiele sind die sogenannten Statusverwaltungsakte, darunter vor allem die Einbürgerung, Beamtenernennung und Approbation, daneben Widmungen, Satzungsgenehmigungen, privatrechtsgestaltende Verwaltungsakte und Vorbescheide, um nur einige zu nennen.

Während die Eintracht des herrschenden dualistischen Ansatzes vordergründig nahezulegen scheint, das Thema habe sich konsolidiert, diagnostiziert die Einleitung zunächst Brüche, die Anlass zu kritischer Nachprüfung geben (S. 2-4). Über den dogmatischen Gehalt des Begriffs der Nebenbestimmungsfeindlichkeit besteht nämlich beträchtliche Unsicherheit. Das zeigt sich bereits an dem ungeklärten Anwendungsbereich eines angeblichen Grundsatzes der Nebenbestimmungsfeindlichkeit, besonders augenfällig an der - im Einzelnen - durchaus verschieden weiten Fallgruppenbildung. Die Unsicherheit setzt sich fort bei den Folgerungen, die sich aus der Nebenbestimmungsfeindlichkeit als Unzulässigkeitsverdikt im Einzelfall ergeben sollen. Aus entwicklungsgeschichtlicher Perspektive wird der Befund prekärer wissenschaftlicher Aufbereitung zum einen aus zivilrechtlicher Stoßrichtung befeuert. Soweit sich die Nebenbestimmungsfeindlichkeit auf die zivilrechtlichen Dogmen der Bedingungs- und Befristungsfeindlichkeit der Gestaltungsrechtsausübung bezieht, rufen neuere kritische Untersuchungen (namentlich Christian Hattenhauer, Einseitige private Rechtsgestaltung, 2011) das Bedürfnis nach verwaltungsrechtswissenschaftlicher Reflexion hervor. Und zum anderen stellt sich die Frage, ob die tradierte Fallgruppenbildung, die in ihrem Kern vielfältige Wandlungen des Fachrechts, die (Teil-)Kodifizierung des Allgemeinen Verwaltungsrechts in den Verwaltungsverfahrensgesetzen, ja mehrfache Systemwechsel der Rechtsordnung bis zur Etablierung des Grundgesetzes über Jahrzehnte hinweg unverändert ,überdauert“ hat, noch den Anforderungen an eine zeitgemäße verwaltungsrechtliche Handlungsformenlehre genügt.

Diesen Forschungsstand aufgreifend, formuliert die Einleitung das zentrale Erkenntnisinteresse der Untersuchung aus: Zum einen die Begriffsbestimmung der Nebenbestimmungsfeindlichkeit anhand der historisch gewachsenen Fallgruppen und des damit in Bezug genommenen Normenbestands, zum anderen die Bestimmung von Grund und Folgen einer Nebenbestimmungsfeindlichkeit (S. 5). Die Perspektive bleibt dabei, wiewohl vor allem in dem referierend-kritischen Teil an den tradierten Fallgruppen orientiert, nicht dem Rechtsakt verhaftet, sondern bezieht ergänzend Erkenntnisse der Rechtsverhältnislehre ein (S. 6 f.). Der methodische Ansatz der Arbeit wird als systematisch, intradisziplinär und dogmengeschichtlich expliziert. Dabei erkennt die Arbeit auch die materiale, d.h. über formal-ordnende Aspekte hinausgehende Systembildung als legitimes Ziel rechtswissenschaftlicher Forschung an (S. 6-11). Die Weiterung der Perspektive um eine intradisziplinäre Dimension trägt den Anleihen Rechnung, die die herrschende Dogmatik beim zivilistischen Nebenbestimmungsrecht nimmt; mit Blick auf die Überlieferung der Nebenbestimmungsfeindlichkeit aus Argumentationsmustern und Ordnungsbildung früherer rechtsgeschichtlicher Epochen bezieht die Arbeit im Sinne eines historisch-informierten Zugriffs zudem dogmengeschichtliche Aspekte ein (S. $11 \mathrm{f}$.). 
II.

Vor diesem Hintergrund skizziert das 1. Kapitel die Grundlagen und Grundbegriffe des Rechts der Nebenbestimmungen zu Verwaltungsakten (S. 15-59). Zunächst wird der Untersuchungsgegenstand auf Verwaltungsakte und ihre Nebenbestimmungen eingegrenzt und es werden die wichtigsten Nebenbestimmungsarten - es handelt sich um die im Katalog des $\S 36$ Abs. 2 VwVfG vertypten - mit ihren arttypischen, in $\S 36$ Abs. 2 VwVfG festgelegten Wirkungen dargestellt (S. 15-25).

Darauf folgt ein knapper Abriss zur Zulässigkeit von Nebenbestimmungen, die gem. $\S \S 1$ Abs. 1 letzter Hs., 36 Abs. 1 Var. 1 VwVfG primär durch besondere Rechtsvorschriften, subsidiär durch $\S 36 \mathrm{VwVfG}$ reguliert wird. Zentral ist insoweit die Unterscheidung zwischen hier sogenannten - sichernden Nebenbestimmungen, die sich darin erschöpfen, die Tatbestandsvoraussetzungen für den Erlass eines (begünstigenden) Verwaltungsakts herbeizuführen (vgl. § 36 Abs. 1 Var. 2 VwVfG), und - hier sogenannten - gestaltenden Nebenbestimmungen, die eine nicht nur rein tatbestandssichernde, sondern darüber hinausgehende gestaltende Funktion einnehmen können (vgl. § 36 Abs. 2 VwVfG) (S. 25-32).

In einem weiteren Schritt schlüsselt die Arbeit den Begriff der Nebenbestimmungsfeindlichkeit auf und verortet diese im System der Fehlergründe (S. 32-58). Die Arbeit zeigt ausgehend von den actus legitimi des Römischen Rechts - eine historische Begriffskontinuität der Nebenbestimmungsfeindlichkeit auf, die ihr einen Selbststand innerhalb der Zulässigkeitsfrage verschafft. Ein Verwaltungsakt ist danach nebenbestimmungsfeindlich, wenn die Beifügung von Nebenbestimmungen an sich, d.h. ungeachtet ihrer Ausgestaltung im Einzelfall, ausgeschlossen ist, wobei ein Unwerturteil über den typischen Regelungsgehalt der Nebenbestimmung getroffen wird (S. 37 f.). Das Unwerturteil tritt in zwei Ausprägungen auf (S. 3841): Einmal als wirkungsbezogener Ausschluss, wenn der Ausschlussgrund sich gerade gegen die arttypische Wirkung bzw. die typische Rechtsfolge der Nebenbestimmung richtet. Als Beispiel kann die spezialgesetzlich abschließend geregelte Aufhebung eines Verwaltungsakts (etwa nach $\S 14$ BBG, $\S 12$ BeamtStG, § 35 StAG) genannt werden, die nicht durch auflösende Nebenbestimmungen (namentlich auflösende Bedingungen und Befristungen sowie Widerrufsvorbehalte) unterlaufen werden darf. Und einmal als inhaltsgerichteter Ausschluss, wenn der gesetzlich festgelegte Inhalt des Verwaltungsakts unverändert bleiben muss, weil die gesetzliche Regelung keine Einschränkung durch Nebenbestimmungen erlaubt. Wichtigstes Beispiel sind gebundene Verwaltungsakte; diese dürfen bei Vorliegen der Erlassvoraussetzungen nicht durch Nebenbestimmungen eingeschränkt werden. Die Arbeit belegt den wirkungsbezogenen Ausschluss mit dem Begriff der Nebenbestimmungsfeindlichkeit im engeren Sinn, den inhaltsgerichteten Ausschluss mit dem Begriff der Nebenbestimmungsfeindlichkeit im weiteren Sinn. Im Unterschied zur Nebenbestimmungsfeindlichkeit im engeren Sinn fällt das Unwerturteil bei der Nebenbestimmungsfeindlichkeit im weiteren Sinn nicht über die typische, durch den Katalog des $\S 36$ Abs. 2 VwVfG festgelegte Nebenbestimmungswirkung bzw. -rechtsfolge, sondern darüber, dass die (einschränkende) Nebenbestimmung als solche zu einer Abweichung vom gesetzlich vorgesehenen Soll-Zustand führt. Das Unwerturteil richtet sich mithin gegen das Nebenbestimmungen eignende Potenzial zur Ausgestaltung des Rechtsverhältnisses. Die so verstandene Nebenbestimmungsfeindlichkeit ist ein Rechtsbegriff (S. 41 f.). Sie knüpft an vorrangige entgegenstehende Rechtsvorschriften an (vgl. § 1 Abs. 1 letzter Hs. VwVfG), die die Beifügung von Nebenbestimmungen an sich ausschließen. Greift deren Ausschlusswirkung ein, entfällt die - komplementäre - Nebenbestimmungstauglichkeit als ungeschriebene tatbestandliche Voraussetzung des $\S 36 \mathrm{VwVfG}$.

Anknüpfend an die differenzierte Begrifflichkeit - Nebenbestimmungsfeindlichkeit im engeren und im weiteren Sinn - macht die Arbeit (typologisch, nicht kategorial) verschiedene Ausschlussgründe aus, indem sie - auch im Hinblick auf die jeweilige Funktion gestaltender und sichernder Nebenbestimmungen - den jeweils unterschiedlichen Zugriff der betreffenden Ausschlussgründe akzentuiert (S. 50-56). Der Nebenbestimmungsfeindlichkeit im engeren 
Sinn liegen - hier sogenannte - echte Verbotsnormen zugrunde. Die Nebenbestimmungen ausschließende Forderung dieser Verbotsnormen ist insoweit absolut. Die Nebenbestimmungsfeindlichkeit im weiteren Sinn hingegen wird durch sogenannte Typisierungsvorschriften statuiert. Der Grad der gesetzlich geforderten Typizität kann dabei von Ausschlussgrund zu Ausschlussgrund verschieden intensiv ausfallen. Besonders relevant ist die durch $\S 36$ Abs. 1 Var. 2 VwVfG eröffnete Möglichkeit der Beifügung von Nebenbestimmungen zur Herstellung der Erlassvoraussetzungen, durch die ein regelmäßig ausreichender, schwächerer Grad der Typenreinheit festgelegt wird.

Die vorstehende Begriffsbildung legt ein in der bisherigen Dogmatik vor allem implizites Verständnis der Nebenbestimmungsfeindlichkeit offen, differenziert dieses aus, markiert die Nebenbestimmungsfeindlichkeit und grenzt sie innerhalb der Zulässigkeitsfrage ab. Mit Blick auf Spezialnormen wie $\S 9$ Abs. 1 S. 1, 2 AufenthG, § 15 Abs. 4 PBefG etc. ließe sich insoweit von einer behutsamen Weiterentwicklung der bisherigen Dogmatik sprechen. Der kritische Punkt der Nebenbestimmungsdogmatik ist demgegenüber, ob Ausschlussgründe im Sinne einer Nebenbestimmungsfeindlichkeit - über punktuelle spezialgesetzliche Vorgaben hinaus - auch für ganze Verwaltungsaktstypen auszumachen sind. Die Frage lautet, anders formuliert, ob der Typus des Verwaltungsakts ein taugliches Kriterium zur Ausweisung der Nebenbestimmungsfeindlichkeit bildet. Damit nähert man sich der Frage nach dem normativen „Sitz“ der Nebenbestimmungsfeindlichkeit, d.h. ob Ausschlussgründe nur in Spezialgesetzen zu verorten oder - generalisierend - auch aus Erfordernissen der Rechtssicherheit abzuleiten sind, die bei bestimmten Arten von Verwaltungsakten besonders stark hervortreten (S. 56$58)$.

III.

Diese Untersuchung, die sich auf die Fallgruppen und Begründungsansätze nebenbestimmungsfeindlicher Verwaltungsakte bezieht, bildet den Gegenstand des 2. Kapitels (S.60187). Die bislang in der Diskussion stehenden Verwaltungsakte werden in - hier sogenannte nebenbestimmungsfeindliche Verwaltungsakte erster und zweiter Ordnung unterteilt. Nebenbestimmungsfeindliche Verwaltungsakte der ersten Ordnung sind danach die übergeordneten Typen der gestaltenden, feststellenden und beurkundenden Verwaltungsakte (S. 60-100). Nebenbestimmungsfeindliche Verwaltungsakte der zweiten Ordnung sind Unterfälle der eben genannten Typen; untersucht werden Statusverwaltungsakte (namentlich Einbürgerung, Beamtenernennung, Approbation), die Widmung, privatrechtsgestaltende Verwaltungsakte (namentlich die Stiftungsanerkennung gem. $§ 80$ BGB), Vorbescheide ( 9 BImSchG) und andere Verwaltungsakte in gestuften Verwaltungsverfahren, streitentscheidende Verwaltungsakte sowie Rechtssatzgenehmigungen (S. 60 f., 101-168). Die Einteilung folgt vor allem Gründen der Übersichtlichkeit, weil die Begründungsansätze für die nebenbestimmungsfeindlichen Verwaltungsakte zweiter Ordnung auf denjenigen für die erster Ordnung aufbauen; insoweit hat die ,Staffelung“ primär abschichtende Funktion. Die Fallgruppen und Begründungsansätze für eine Nebenbestimmungsfeindlichkeit werden - unter enger Anbindung an den damit in Bezug genommenen (fachrechtlichen) Normenbestand - auf ihre Tragfähigkeit im geltenden Recht untersucht. Fachrechtlich bleiben Nebenbestimmungen innerhalb der Grenzen etwaiger Formbindung und sonstiger Spezialvorschriften grundsätzlich zulässig; die Reichweite des Nebenbestimmungsausschlusses betrifft insoweit Fragen der Auslegung und Anwendung der spezialgesetzlichen Ausschlussgründe. Eine weiter ausgreifende Nebenbestimmungsfeindlichkeit, wie sie die herrschende Lehre vielfach für die genannten Verwaltungsakte ,an sich“ annimmt, kann aus dem Fachrecht nicht abgeleitet werden; insoweit müsste man andere normative Grundlagen bemühen.

Beschränkt man sich auf das Wesentliche, stützt sich die aktzentrierte Nebenbestimmungsfeindlichkeit traditioneller Lehre - gröber verallgemeinernd - auf folgende Gesichtspunkte: Erstens - vor allem für Bedingungen und Befristungen - auf die Erfordernisse der 
Rechtssicherheit, zweitens - vor allem für Auflagen - das Akzessorietätsprinzip und drittens pauschal - die Berufung auf die Natur der Sache.

Zur Rechtssicherheits-Argumentation: Bei den genannten Fallgruppen sollen Bedürfnisse der Rechtssicherheit die Beifügung von Nebenbestimmungen ausschließen. Diese Behauptung steht unverkennbar in der Tradition der Gestaltungsrechtsdogmatik mit den Dogmen der Bedingungs- und Befristungsfeindlichkeit. Allerdings kommt eine normative Anknüpfung an die zivilrechtliche Norm des $\S 388$ S. 2 BGB für Verwaltungsakte nicht in Betracht. Denn die Voraussetzungen für eine analoge Anwendung fehlen (S. 66-68); es greifen letztlich dieselben Gesichtspunkte durch, die auch einem auf das Rechtsstaatsprinzip (Artt. 20 Abs. 3, 28 Abs. 1 GG) gestützten Ausschluss entgegenstehen: Das Rechtsstaatsprinzip erweist sich als zu grobmaschig, um Nebenbestimmungen bei ganzen Typen von Verwaltungsakten pauschal einem Unzulässigkeitsverdikt zu unterwerfen. Durch Nebenbestimmungen hervorgerufene Schwebezustände können vielmehr im Rahmen eines konkreten Verwaltungsrechtsverhältnisses eingehegt werden; zentral sind insoweit insbesondere einseitig-hoheitliche Feststellungsmöglichkeiten der Verwaltung (S. 74-77). Anlass zu einer abweichenden Beurteilung besteht auch dann nicht, wenn mit dem Verwaltungsakt besondere Stabilitätserwartungen verbunden werden. Denn auch Verwaltungsakte, die mit Nebenbestimmungen erlassen werden, gewähren dem Adressaten im Rahmen ihres Regelungsgehalts eine gesicherte Rechtsposition (S. 178-180).

Zum Akzessorietätsprinzip: Dieser Argumentationsstrang wendet sich vor allem gegen die Zulässigkeit von Auflagen. Eine Auflage dürfe nur belastend wirken, wenn ein Verhalten gestattet wird, und auch erst nach Gebrauchmachen von der Begünstigung. Andernfalls würde der Bürger vorzeitig, insbesondere entgegen dem Akzessorietätsprinzip belastet. Das soll Auflagen bei nicht vollziehungsbedürftigen Verwaltungsakten, namentlich gestaltenden und feststellenden, ausschließen. Dagegen spricht, dass eine Auflage erst bestandskräftig werden muss, ehe sie vollzogen werden darf (vgl. § 6 Abs. 1 VwVG). Im Übrigen kann sich der Adressat gegen eine belastende Auflage mit der Anfechtungsklage wehren. Er wird also nicht unzumutbar belastet, insbesondere nicht stärker als auch sonst bei der Beifügung von Nebenbestimmungen (S. 78-80).

Zur Argumentation aus der Natur der Sache: Die Natur der Sache soll der Beifügung von Nebenbestimmungen insbesondere bei Statusverwaltungsakten entgegenstehen. Dieser Begründungsansatz verschleiert indes mehr, als dass er aufhellt. Forderungen aus der Natur der Sache stellen in erster Linie Gestaltungsempfehlungen an den Gesetzgeber dar. Lässt der Gesetzgeber insoweit eine Lücke, hat die Forderung aus der Natur der Sache lediglich Leitliniencharakter. Die Natur der Sache kommt bei der Entscheidung über Nebenbestimmungen folglich über Zweckmäßigkeitserwägungen zum Tragen. Zu dieser Zweckmäßigkeitsprüfung wiederum ist an erster Stelle die Verwaltung im Rahmen ihrer Ermessensausübung, die jeder Nebenbestimmungsbeifügung nach $\S 36$ VwVfG vorausliegt, berufen. Die Frage lautet dann nicht: Sind Nebenbestimmungen ausgeschlossen?, sondern vielmehr: Ist die Beifügung einer Nebenbestimmung zweckmäßig (S. 175-178)?

Im Rahmen einer zusammenführenden Betrachtung werden am Ende des 2. Kapitels noch einmal die Gesichtspunkte dargestellt, die gegen eine am Verwaltungsaktstyp festzumachende Nebenbestimmungsfeindlichkeit traditioneller Lehre sprechen: Nämlich dass die überkommenen begriffshistorischen, ontologischen und funktionalen Argumente methodologisch keine tragfähige Basis dafür bilden bzw. keine hinreichende normative Anknüpfung finden, um ganze Typen von Verwaltungsakten als nebenbestimmungsfeindlich auszuweisen (S. 169-180); eine Nebenbestimmungsfeindlichkeit der genannten Verwaltungsaktstypen lässt sich insbesondere nicht unter Berufung auf allgemeine Rechtsgrundsätze und Rechtsgedanken oder Gewohnheitsrecht begründen (S. 180-182). Die Kritik am traditionellen typengebundenen Ansatz schließt mit der Feststellung, dass die herkömmliche Fallgruppenbildung zu einer diffusen und inkonsistenten Kasuistik führt, die Rechtsfindung verunklart und den Blick vom 
maßgeblichen Gesetz ablenkt (S. 186). Die Arbeit plädiert demgegenüber für eine am Spezialgesetz ausgerichtete Dogmatik, die in den Ordnungsrahmen des Rechtsverhältnisses eingespannt werden soll.

IV.

Diesen Ansatz - die Grundlegung eines normzentrierten Verständnisses nebenbestimmungsfeindlicher Verwaltungsakte - entwickelt die Arbeit im 3. Kapitel näher (S. 188-243). Zunächst wird die Annahme einer grundsätzlichen Nebenbestimmungstauglichkeit aller materiellen Verwaltungsakte ungeachtet ihres Typs legitimatorisch auf die Eigenständigkeit der Verwaltung als verfassungsrechtlichem Tatbestand (Art. 20 Abs. 2 S. 2, Abs. 3 GG) zurückgeführt; einfachrechtlich heruntergebrochen verbürgt das gesetzliche Ermessen gem. § 36 VwVfG bei der Entscheidung über die Beifügung von Nebenbestimmungen eine verfassungsrechtlich fundierte In-dubio-Regel für die Zulässigkeit von Nebenbestimmungen (S. 188192). Die Beifügung ist ausgeschlossen, insoweit das Ermessen gesetzlich begrenzt ist.

Maßgebliches Ordnungskriterium ist das Spezialgesetz, wobei der zugrunde liegende Gesetzesbegriff über das Fachrecht hinausreicht und grundsätzlich alle materiellen Gesetze erfasst (S. 199-203). Die Arbeit stellt in einem Überblick sodann - die Begrifflichkeiten aus dem 1. Kapitel wieder aufgreifend - Beispiele für (inhaltsgerichtete) Typisierungsnormen dar, die der Beifügung von Nebenbestimmungen im Sinne einer Nebenbestimmungsfeindlichkeit (im weiteren Sinne) entgegenstehen (S. 203-208). Solche Typisierungen lassen sich sowohl im Verfassungsrecht (z.B. Art. 33 Abs. 2 GG, Art. 16 Abs. 1 GG) als auch auf einfachgesetzlicher Ebene nachweisen (z.B. § 3 Abs. 1 S. 1 B ̈̈O, § 9 Abs. 1 S. 1, 2 AufenthG). Beispiele für (wirkungsbezogene) Verbotsnormen, in denen sich eine Nebenbestimmungsfeindlichkeit im engeren Sinn ausdrückt, wiederum sind namentlich spezialgesetzliche Vorschriften über die Aufhebung von Verwaltungsakten (etwa § 14 BBG, § 12 BeamtStG, § 35 StAG) (S. 208211). Die Reichweite des Ausschlusses ist dabei stets eine Frage der Auslegung der betreffenden Norm. Im Falle einer Typisierungsnorm ist der von dieser geforderte Grad an Typizität zu ermitteln (der z.B. bei begünstigenden Verwaltungsakten der gebundenen Verwaltung grundsätzlich nach Maßgabe des $§ 36$ Abs. 1 Var. 2 VwVfG gelockert ist). Im Falle einer Verbotsnorm kommt es maßgeblich darauf an, ob sich der Ausschlussgrund nach Sinn und Zweck gerade gegen die spezifische Rechtswirkung eines Nebenbestimmungstyps richtet. Das kann im Einzelfall eine Anwendung der Ausschlussnorm über ihren originären Anwendungsbereich hinaus im Wege der Analogie erforderlich machen, umgekehrt aber auch die einschränkende Auslegung oder teleologische Reduktion einer zu weit gefassten Ausschlussnorm. In besonderer Weise zeigen sich die Auswirkungen des normbezogenen Ansatzes am Beispiel der privatrechtsgestaltenden Verwaltungsakte (S. 211-231): Hier wirken für das Privatrechtsgeschäft statuierte Bedingungsverbote über die öffentlich-rechtliche Befugnisnorm als Ermessensbindungen auf die behördliche Entscheidung ein; Nebenbestimmungen sind insoweit ausgeschlossen, wie der Zweck der privaten Verbotsnorm reicht (S. 223). Umgekehrt kann im Einzelfall eine teleologische Reduktion öffentlich-rechtlicher Nebenbestimmungsverbote im Sinne einer ,privatrechtsorientierten“ Auslegung angezeigt sein, wenn die Sicherheit des Privatrechtsverkehrs einen Nebenbestimmungsausschluss nicht gebietet (und keine anderen Gesichtspunkte für eine Anordnung des Ausschlusses ersichtlich sind).

Das 3. Kapitel stellt in seinem letzten Abschnitt (S. 231-243) zunächst das Flexibilisierungspotenzial einer normzentrierten Dogmatik gegenüber der bisherigen aktzentrierten Dogmatik dar. Dieses hängt wiederum maßgeblich davon ab, ob die gegenüber dem aktzentrierten Ansatz „freigesetzten“ Nebenbestimmungen ihr Verwirklichungspotenzial einlösen können und so nicht nur nominell, sondern auch tatsächlich eine spürbare Weiterung des $\mathrm{Ne}$ benbestimmungsinstrumentariums zu Buche schlägt. Als Ergebnis hält die Arbeit fest, dass mit dem normbezogenen Ansatz nicht nur eine beträchtliche Flexibilisierung der Handlungsmöglichkeiten der Verwaltung gegenüber dem bisherigen, aktzentrierten Ansatz einhergeht, sondern diesem auch ein erheblicher Zuwachs an Rechtssicherheit gegenübersteht: Der Ne- 
benbestimmungsausschluss hängt von punktuell entgegenstehenden Vorschriften ab, wobei die Orientierung an den Gruppen der Typisierungs- und Verbotsnormen einerseits und die Auslotung der Potenziale des Rechtsverhältnisses andererseits die Bestimmung des zulässigen und tauglichen Wirkbereichs einer Nebenbestimmungen erleichtert und die Behörde dadurch zur zielgenauen und gesetzeskonformen Ausgestaltung der Nebenbestimmung diszipliniert (S. 243).

V.

Wenn mit einer im Einzelfall aufzuweisenden Nebenbestimmungsfeindlichkeit ein gesetzliches Unzulässigkeitsurteil einhergeht, ist die Frage aufgeworfen, welche Rechtsfolgen sich an das Unzulässigkeitsurteil anschließen. Dieses Themas nimmt sich das 4. Kapitel - Fehlerfolgen nebenbestimmungsfeindlicher Verwaltungsakte auf Grundlage eines normzentrierten Ansatzes - an (S. 244-282). Unter dem traditionellen, aktzentrierten Ansatz bleibt häufig unklar, welche Fehlerfolgen sich aus der Nebenbestimmungsfeindlichkeit ergeben sollen; das Spektrum der vorgeschlagenen Lösungen changiert seit alters von (bloßer) Anfechtbarkeit oder Teilnichtigkeit bzw. Streichung der Nebenbestimmung bis hin zur Gesamtnichtigkeit des Verwaltungsakts, wobei die normative Anknüpfung sowohl des Unzulässigkeitsurteils als solchem als auch der maßgeblichen Fehlerfolgenregelung vielfach offen bleibt und im Übrigen hierüber, soweit ersichtlich, noch keine Einigkeit erzielt werden konnte (S. 244-246). Demgegenüber führt der hier vorgeschlagene normzentrierte Ansatz über das Spezialgesetz zur Anwendung etwaiger fachrechtlicher, besonderer Fehlerfolgenregelungen, subsidiär zur Anwendung des allgemeinen Fehlerfolgenrechts. Das macht zunächst eine Auseinandersetzung mit dem Verhältnis von Gesamtverwaltungsakt und Nebenbestimmung in der Rechtswidrigkeitsperspektive, mithin eine Positionierung in der Diskussion um den Gegenstand des Rechtswidrigkeitsurteils (Gesamtverwaltungsakt oder Nebenbestimmung) und die Rechtswidrigkeitsfolgen (auf Gesamtverwaltungsakt zu beziehen oder gegebenenfalls auf Teile des Verwaltungsakts zu beschränken), insbesondere die Frage der Teilbarkeit der Nebenbestimmungen vom Restverwaltungsakt, erforderlich (S. 244-272). Die Arbeit gelangt nach einer Auseinandersetzung mit den hierzu in Literatur und Rechtsprechung vertretenen Ansichten zu der Auffassung, dass eine unzulässige Nebenbestimmung zur Rechtswidrigkeit des (Gesamt-)Verwaltungsakts führt (S. 247 f.). Die Rechtswidrigkeitsfolgen allerdings können auf Teile des Verwaltungsakts beschränkt bleiben. Der in der Beifügung einer Nebenbestimmung entgegen einem Ausschlussgrund liegende Fehler wird unter den Voraussetzungen des $§ 44$ Abs. 4 VwVfG, der über seinen originären Anwendungsbereich hinaus auch Maßstabsnorm für die behördliche oder gerichtliche Teilaufhebung eines (auch nur) rechtswidrigen Verwaltungsakts ist, abhängig von der Schwere des Fehlers durch Teilaufhebung oder durch gesetzliche Anordnung der Teilnichtigkeit sanktioniert (S. 249-272). Fehlt es an den Voraussetzungen des $\S 44$ Abs. 4 VwVfG, kommt eine Teilaufhebung oder -nichtigkeit indessen nicht in Betracht. Liegt ein besonders schwerer Fehler und damit ein Nichtigkeitsgrund vor, führt dies zur Gesamtnichtigkeit des Verwaltungsakts; bloße Rechtswidrigkeit ist durch Aufhebung des Gesamtverwaltungsakts zu sanktionieren.

Verstößt die Beifügung einer Nebenbestimmung gegen eine Verbots- oder Typisierungsnorm im oben genannten Sinne, kann, je nach Fehlerschwere und Offenkundigkeit - beide liegen umso näher, je deutlicher der Ausschlussgrund aus dem Gesetz hervorgeht - ein Nichtigkeitsgrund gem. § 44 Abs. 1 VwVfG verwirklicht sein, wobei sich die Nichtigkeitsfolge gegebenenfalls auf die Nebenbestimmung beschränkt ( $\$ 44$ Abs. 4 VwVfG). Unterhalb der Schwelle der besonderen Fehlerschwere bleibt es bei der bloßen Rechtswidrigkeit des Verwaltungsakts, der gegebenenfalls - unter den Voraussetzungen des entsprechend anwendbaren $§ 44$ Abs. 4 VwVfG - durch Teilaufhebung begegnet werden kann (S. 272-282). 
VI.

Die Schlussbetrachtung bringt die Grundlagen einer normzentrierten Dogmatik nebenbestimmungsfeindlicher Verwaltungsakte begrifflich, normativ und methodologisch auf den Punkt und stellt die wesentlichen systematischen und praktischen Erträge der Arbeit überblicksartig dar (S. 283-294). Entgegen der bisherigen Dogmatik ist der Typus des Verwaltungsakts kein taugliches Kriterium zur Ausweisung einer Nebenbestimmungsfeindlichkeit. Allein maßgeblich ist vielmehr der - nicht über den Typus des Verwaltungsakts vermittelte Rückgriff auf das Gesetz, wobei der Gesetzesbegriff über das Fachrecht hinausreicht und grundsätzlich alle materiellen Gesetze erfasst. Der Fokus auf das Spezialgesetz führt zu einer präziseren Begrifflichkeit: Verbotsnormen mit ihrem absoluten, auf Verhütung der spezifischen Rechtsfolgen einer Nebenbestimmung gerichteten Ausschussgehalt sind komplementär zur Nebenbestimmungsfeindlichkeit im engeren Sinn, Typisierungsnormen mit ihrem relativen, gegen die ausgestaltende Funktion von Nebenbestimmungen gerichteten Ausschlussgehalt sind komplementär zur Nebenbestimmungsfeindlichkeit im weiteren Sinn. Mit der Ausrichtung auf das Spezialgesetz geht auch eine Schärfung des Anwendungsbereichs der Nebenbestimmungsfeindlichkeit einher: Der Nebenbestimmungsausschluss beantwortet sich maßgeblich nach einer an Sinn und Zweck orientierten Auslegung des gesetzlichen Ausschlussgrunds. Die Anknüpfung der Nebenbestimmungsfeindlichkeit an das Spezialgesetz führt damit auch für die Fehlerfolgen nebenbestimmungsfeindlicher Verwaltungsakte in gewohnte Bahnen: Die Fehlerfolgen beurteilen sich nach allgemeinen Grundsätzen. Im Ergebnis bedeutet die Ausrichtung der Dogmatik nebenbestimmungsfeindlicher Verwaltungsakte auf das Spezialgesetz nicht nur eine erhebliche Flexibilisierung des Gestaltungspotenzials der Verwaltung, sondern auch einen beträchtlichen Zuwachs an Rechtssicherheit. Sie schafft für den Rechtsanwender Orientierungspunkte, insbesondere im Hinblick auf die nach bisheriger Dogmatik häufig offene Frage, ob und welche Nebenbestimmungen in welchem Umfang und mit welchen Folgen ausgeschlossen sind. Den normzentrierten Ansatz charakterisiert die Arbeit abschließend als Beispiel für eine eigenständige Verwaltung in einer einheitlichen Rechtsordnung (S. 294). 